\title{
A visual interaction consensus model for social network group decision making with trust propagation
}

\author{
Jian $\mathrm{Wu}^{\mathrm{a}, \mathrm{b}}$, Francisco Chiclana ${ }^{\mathrm{b}}$, Hamido Fujita ${ }^{\mathrm{c}}$, Enrique Herrera-Viedmad,e $^{\mathrm{d}, \mathrm{e}}$ \\ ${ }^{a}$ School of Economics and Management, Shanghai Maritime University, Shanghai 201306, China \\ ${ }^{b}$ Centre for Computational Intelligence, Faculty of Technology, De Montfort University, Leicester, UK \\ ${ }^{c}$ Iwate Prefectural University, Takizawa, Iwate, Japan \\ ${ }^{d}$ Department of Computer Science and Artificial Intelligence, University of Granada, Granada, Spain \\ ${ }^{e}$ Department of Electrical and Computer Engineering, Faculty of Engineering, King Abdulaziz University, Jeddah \\ 21589, Saudi Arabia
}

\begin{abstract}
A theoretical visual interaction framework to model consensus in social network group decision making (SN-GDM) is put forward with following three main components: (1) construction of trust relationship; (2) trust based recommendation mechanism; and (3) visual adoption mechanism. To do that, dual trust propagation is investigated to connect incomplete trust relationship by trusted third partners, in a way that it can fit our intuition in these cases: trust values decrease while distrust values increase. Trust relationship is proposed to be used in determining the trust degree of experts and in aggregating individual opinions into a collective one. Three levels of consensus degree are defined and used to identify the inconsistent experts. A trust based recommendation mechanism is developed to generate advices according to individual trust relationship, making recommendations more likeable to be implemented by the inconsistent experts to achieve higher levels of consensus. Therefore, it has an advantage with respect to existing interaction models because it does not force the inconsistent experts to accept advices irrespective of their trust on them. Finally, a visual adoption mechanism, which provides visual information representations on experts' individual consensus positions before and after adopting the recommendation advices, is presented and analysed theoretically. Experts can select their appropriate feedback parameters to achieve a balance between group consensus and individual independence. Consequently, the proposed visual interaction model adds real and needed flexibility in guiding the consensus reaching process in SN-GDM.
\end{abstract}

Keywords: Social network group decision making, Visual interaction, Consensus, Trust recommendation, Adoption mechanism, Trust propagation

\section{Introduction}

In group decision making (GDM), a group of experts express preference values (opinions) on alternatives and interact to derive a common solution $[14,22]$. Group experts usually come from

Email addresses: jyajian@163.com (Jian Wu), chiclana@dmu.ac.uk (Francisco Chiclana), HFujita-799@acm.org (Hamido Fujita), viedma@decsai.ugr.es (Enrique Herrera-Viedma) 
multiple organisations with different backgrounds and knowledge on the decision making problem faced, which may lead to inconsistency problems among the group [5, 29, 30, 35, 36, 39, 43, 48]. Thus, how to reach consensus is relevant and a hot topic that has attracted the interest of researchers in the field of GDM $[2,12,17,42]$. The group interaction consensus model has been proved to be an effective method to increase consensus within a group of experts because it supports inconsistent experts, i.e. experts with a consensus level below a target threshold value, with advices on how to modify their preference values [23, 47]. However, these known group interaction consensus models do not take into account the trust relationship among the experts in the group [14, 20,44], which makes them not suitable for GDM problems in a social network framework where the relationships between the members of a group is present and should be considered in obtaining a solution of consensus $[13,28,33]$.

A new trend of GDM today is that individuals rely on the opinions and social appraisal support from their close friends or people with similar interests, and then the social relationship should be taken into account in the interaction process of GDM $[1,19,21,24,25,27]$. An example is the community review website Ciao (www.ciao.co.uk), where users can write reviews about consumer products and assign a rating to the products and the reviews written by others. In essence, Ciao is a social network, i.e., "an association of people drawn together by family, work or hobby". By reviewing and talking, consumers can build a trust relationship between them, which in turn can be used to reach higher consensus level in the group interaction. Then, the trust relationship should be considered an important factor influencing the consensus process in GDM as pointed by Herrera-Viedma et al. [16]. This social network group decision making (SN-GDM) would be a completely new type of decision making because it takes into account the trust relationship in the process of reaching consensus while the traditional GDM models neglect it. Consequently, key issues in SN-GDM problems would be: (1) the study and modelling of trust relationship between experts, and (2) how to reach group consensus under trust relationship.

In a realistic trust network, there exist indirect trust relationships between experts that are unknown to each other as Fig. 1 illustrates. For such cases, there is a need to devise a mechanism to find out whether or not an unknown expert can be trusted [38]. To do that, a trust chain via trusted third partners (TTPs) can be built to propagate trust to an unknown expert. This will obviously require the development of appropriate trust propagation operators. Victor et al. [31] have proposed t-norms to propagate trust and t-conorms to propagate distrust, while $\mathrm{Wu}$ et al. [41] developed a uninorm operator to propagate both trust and distrust at the same time. However, these operators share the limitation of decreasing both the trust and distrust values simultaneously, which conflicts with our intuition because the trust propagating process using trusted third parters (TTP) may produce information attenuation that makes trust values to decrease but distrust values to increase. To avoid this issue, this article will investigate a novel dual trust propagation operator based on the t-norm Einstein 
product and the t-conorm Einstein sum. In addition, its desirable properties including 'T-subnorm' and 'T-upconorm' can be guaranteed.

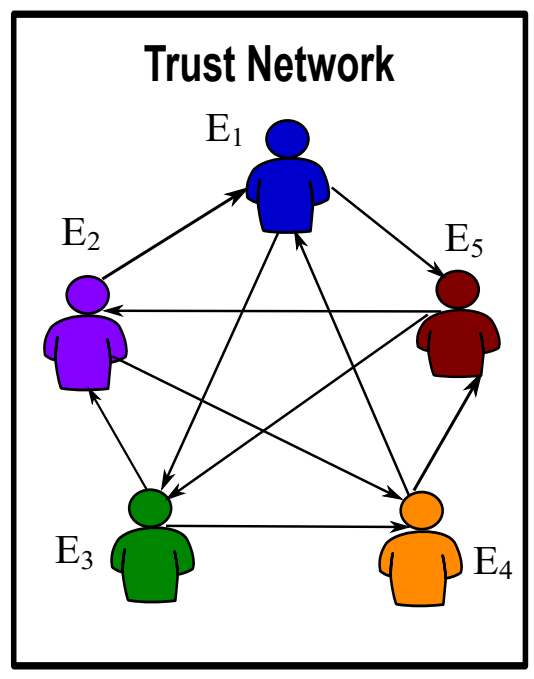

Figure 1: Trust Network: solid arrows mean presence of direct trust

As aforementioned, the group interaction models with a recommendation mechanism were regarded as an effective method to reach satisfied consensus level in traditional GDM [14, 44]. However, they usually generate recommendation advices for the inconsistent experts based on the arithmetic average of the individual opinions of the experts in the group with no consideration at all on their trust relationship. As such, in the known group interaction models inconsistent experts have no idea whether the change of opinion recommendation advice given can be trusted/distrusted. This is not ideal and it is indeed in conflict with real decision making cases because it is up to the experts to adopt or not the recommendation advices provided to him/her [9, 34]. Additionally, known group interaction models share another limitation associated to the fixation of a feedback parameter, which decreases the adaptability of the recommendation advices to inconsistent experts' willingness of change their individuals original opinions. Therefore, they might wish to keep unaltered their independence $[3,7$, $37,40]$ or, if possible, to reduce changes cost $[6,8,10,11]$. In other words, experts are willing to keep balance between group consensus and individual independence.

To resolve the aforementioned issues, this article firstly investigates a trust based recommendation mechanism to generate recommendation advices by trust relationship obtained via TTPs. This will surely contribute to inconsistent experts willingness to adopt or implement the advices received. Secondly, a visual adoption mechanism in which the consensus status is simulated by choosing different feedback parameter is developed. The visual representations of information can help experts to analyse their consensus position before and after adopting the advices received. Consequently, the proposed visual group interaction model will allow experts to decide by themselves on selecting the right feedback parameter to balance consensus and independence. Obviously, this new approach offers more flexibility than the known group interaction models, and so it is suitable for SN-GDM contexts. 
The rest of this paper is set out as follows: Section 2 introduces the definition of dual trust propagation operator and studies its desirable properties. The complete trust relationship of group experts is also constructed in this section. In Section 3, the concept of trust degree (TD) is defined and used to aggregate the individual trust decision making matrices into a collective one. Section 4 presents the novel visual interaction model for consensus in SN-GDM. In detail, it includes the trust based recommendation mechanism and the visual adoption mechanism. The framework of the visual interaction consensus model proposed including all steps of the decision process is provided in Section 5. Section 6 uses a numerical example to illustrate the proposed methods. Finally, conclusions are drawn in Section 7.

\section{Dual Trust Propagation and Aggregation in Social Network}

Social Network Analysis (SNA) [13, 28, 33] studies the relationships between social entities like members of a group, corporations or nations. Therefore, it enables us to examine the structural and locational properties including centrality, prestige, structural balance, trust relationship etc. There are three notational schemes in SNA analysis:

- Graph, as in Fig. 1, where the network is viewed as a set of nodes joined by lines (edges).

- Algebraic, which allows to distinguish several distinct relations and to represent combinations of relations. The corresponding algebraic representation of Fig. 1 would be:

$$
\begin{array}{lllll}
E_{1} R E_{3} & E_{3} R E_{4} & E_{4} R E_{5} & E_{5} R E_{2} & E_{2} R E_{4} \\
E_{4} R E_{1} & E_{1} R E_{5} & E_{5} R E_{3} & E_{3} R E_{2} & E_{2} R E_{1}
\end{array}
$$

where $E_{i} R E_{j}$ represents the existence of a directed graph edge from node $E_{i}$ to node $E_{j}$.

- Sociometric, which presents relational data in a two-ways matrix called sociomatrix. The corresponding sociomatrix representation of Fig. 1 would be:

$$
\left(\begin{array}{lllll}
0 & 0 & 1 & 0 & 1 \\
1 & 0 & 0 & 1 & 0 \\
0 & 1 & 0 & 1 & 0 \\
1 & 0 & 0 & 0 & 1 \\
0 & 1 & 1 & 0 & 0
\end{array}\right)
$$

The above sociomatrix represents a binary complete relation. This type of sociomatrix has two main limitations:

1. It is not suitable to model uncertainty associated to the relationship representation in social network as it is the case with trust, which is indeed often interpreted as a gradual phenomenon: humans do not merely reason in terms of 'trusting' and 'not trusting', but rather trusting someone 'very much' or 'more or less' [4]. 
2. Binary trust relationships cannot be propagated and as such indirect trust relationships cannot be built.

To overcome these problems, this article adopts one type of social networks, namely trust network in which the users explicitly express their opinion as trust and distrust statements [32].

Definition 1 (Trust Function (TF)). A tuple of the type $\lambda=(t, d)$ where $t, d \in[0,1]$, in which the first component $t$ is a trust degree, and the second component $d$ is a distrust degree will be referred to as a trust function value. The set of trust function values (TFs), or trust function, will be denoted by $\Lambda=\{\lambda=(t, d) \mid t, d \in[0,1]\} \equiv[0,1]^{2}$.

The concepts of trust score and knowledge degree were proposed to define an order relation in the set of TFS [41]:

Definition 2 (Trust Score (TS)). The trust score is a mapping on the set of TFs, $\Lambda$, that associates a value in $[0,1]$ to each trust function value $\lambda$ as follows:

$$
\begin{gathered}
T S: \Lambda \longrightarrow[0,1] \\
T S(\lambda)=\frac{t-d+1}{2}
\end{gathered}
$$

Definition 3 (Knowledge Degree (KD)). The knowledge degree is a mapping on the set of TFs, $\Lambda$, that associates a value in $[0,1]$ to each trust function value $\lambda$ as follows:

$$
\begin{gathered}
K D: \Lambda \longrightarrow[0,1] \\
K D(\lambda)=(1-t-d)^{2}
\end{gathered}
$$

Definition 4 (Order Relation of TFs). Given two TFs, $\lambda_{1}$ and $\lambda_{2}, \lambda_{1}$ precedes $\lambda_{2}$

$$
\lambda_{1} \prec \lambda_{2}
$$

if and only if one of the following conditions is true:

1. $T S\left(\lambda_{1}\right)<T S\left(\lambda_{2}\right)$

2. $T S\left(\lambda_{1}\right)=T S\left(\lambda_{2}\right) \wedge K D\left(\lambda_{1}\right)>K D\left(\lambda_{2}\right)$

The above order relation of TFs can be used to distinguish the most trusted expert from the group, which in turn can be useful in performing an heterogeneous aggregation of individual opinions by establishing the ordering of the decision matrix values to be aggregated. 


\subsection{Trust propagation}

Trust propagation operators have been proposed and built using the general concepts of the triangular norms and triangular conorms. A function $T:[0,1]^{2} \rightarrow[0,1]$ is called a triangular norm (t-norm for short) if and only if it is commutative, associative, monotonic and satisfies the following boundary conditions $T(x, 1)=x, \forall x$. A function $S:[0,1]^{2} \rightarrow[0,1]$ is called a triangular conorm (t-conorm for short) if and only if it is commutative, associative, monotonic and satisfies the following boundary conditions $S(x, 0)=x, \forall x$. In the following, we will be making use of the Einstein product, $\otimes_{\varepsilon}$, and Einstein sum, $\oplus_{\varepsilon}$ as the t-norm and a t-conorm [18], respectively, to illustrate the general approach to trust propagation proposed in this paper:

$$
\begin{gathered}
E_{\otimes}=a \otimes_{\varepsilon} b=\frac{a \cdot b}{1+(1-a) \cdot(1-b)}, \forall(a, b) \in[0,1]^{2} \\
E_{\oplus}=a \oplus_{\varepsilon} b=\frac{a+b}{1+a \cdot b}
\end{gathered}
$$

It is worth mentioning that the greatest of all t-norms is the minimum operator, while the lowest of all t-conorm is the maximum operator. Consequently, we have that

$$
\begin{gathered}
E_{\otimes}\left(x_{1}, x_{2}\right) \leq \min \left\{x_{1}, x_{2}\right\}(\text { t-subnorm }) \\
\max \left\{x_{1}, x_{2}\right\} \leq E_{\oplus}\left(x_{1}, x_{2}\right)(\text { t-upconorm })
\end{gathered}
$$

The above definitions just present the $E_{\oplus}$ and $E_{\otimes}$ as aggregation operators of two arguments. However, the associativity property allows their use with $n$ arguments:

$$
E_{\oplus}\left(x_{1}, \ldots, x_{n}\right)=\frac{\prod_{i=1}^{n}\left(1+x_{i}\right)-\prod_{i=1}^{n}\left(1-x_{i}\right)}{\prod_{i=1}^{n}\left(1+x_{i}\right)+\prod_{i=1}^{n}\left(1-x_{i}\right)}
$$

and

$$
E_{\otimes}\left(x_{1}, \ldots, x_{n}\right)=\frac{2 \prod_{i=1}^{n} x_{i}}{\prod_{i=1}^{n}\left(2-x_{i}\right)+\prod_{i=1}^{n} x_{i}}
$$

The monotonocity property implies that the t-subnorm and t-upconorm properties also hold for the case on of $n$ arguments:

$$
\begin{aligned}
& E_{\otimes}\left(x_{1}, x_{2}, \ldots x_{n}\right) \leq \min \left(x_{1}, x_{2}, \ldots x_{n}\right) \\
& \max \left(x_{1}, x_{2}, \ldots x_{n}\right) \leq E_{\oplus}\left(x_{1}, x_{2}, \ldots x_{n}\right)
\end{aligned}
$$

Existing trust propagation operators do not take into account the distrust value of TTP in the trust chain [31, 41]; additionally they also have one main limitation in that both trust and distrust values decrease simultaneously. Considering the information attenuation in a realistic trust propagating process via a TTP, a reasonable policy should be that the trust value decreases and the distrust value increases as a result of a propagation process. To achieve this aim, we propose a dual trust propagation operator by combining the Einstein sum operator $E_{\oplus}$ and the Einstein product operator $E_{\otimes}$ (see Fig. $2)$. 


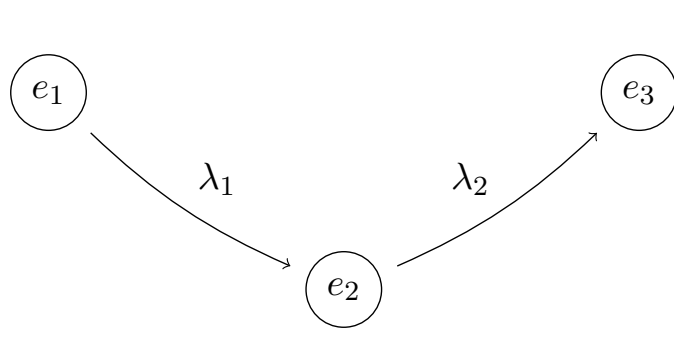

(a) No direct TF between $e_{1}$ and $e_{3}$

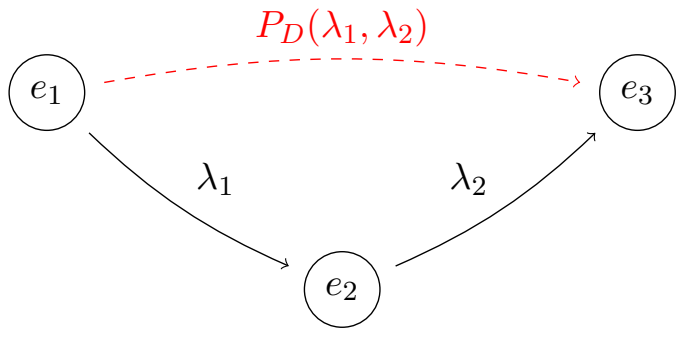

(b) Trust propagation between $e_{1}$ and $e_{3}$ via $e_{2}$

Figure 2: Dual trust propagation via indirect chain of TFs

Definition 5. Let $\Lambda$ be the set of TFs. The dual trust propagation operator $P_{D}$ is mapping, $P_{D}$ : $\Lambda \times \Lambda \rightarrow \Lambda$, that associates to two TFs $\lambda_{1}=\left(t_{1}, d_{1}\right), \lambda_{2}=\left(t_{2}, d_{2}\right)$, the following TF output

$$
P_{D}\left(\lambda_{1}, \lambda_{2}\right)=\left(E_{\otimes}\left(t_{1}, t_{2}\right), E_{\oplus}\left(d_{1}, d_{2}\right)\right)=\left(\frac{t_{1} t_{2}}{1+\left(1-t_{1}\right)\left(1-t_{2}\right)}, \frac{d_{1}+d_{2}}{1+d_{1} \cdot d_{2}}\right)
$$

The following observations are noticed:

1. Because $E_{\otimes}\left(t_{1}, t_{2}\right) \leq \min \left\{t_{1}, t_{2}\right\}$ and $\max \left\{d_{1}, d_{2}\right\} \leq E_{\oplus}\left(d_{1}, d_{2}\right)$, the dual trust propagation operator $P_{D}$ verifies that the trust value and distrust value decreases and increases, respectively, as a result of the propagation process.

2. $P_{D}$ inherits the commutativity and associativity properties of $E_{\oplus}$ and $E_{\otimes}$.

\section{Commutativity:}

$$
P_{D}\left(\lambda_{2}, \lambda_{1}\right)=\left(E_{\otimes}\left(t_{2}, t_{1}\right), E_{\oplus}\left(d_{2}, d_{1}\right)\right)=\left(E_{\otimes}\left(t_{1}, t_{2}\right), E_{\oplus}\left(d_{1}, d_{2}\right)\right)=P_{D}\left(\lambda_{1}, \lambda_{2}\right)
$$

\section{Associativity:}

$$
\begin{aligned}
P_{D}\left[P_{D}\left(\lambda_{1}, \lambda_{2}\right), \lambda_{3}\right] & =P_{D}\left[\left(E_{\otimes}\left(t_{1}, t_{2}\right), E_{\oplus}\left(d_{1}, d_{2}\right)\right),\left(t_{3}, d_{3}\right)\right]=\left(E_{\otimes}\left(E_{\otimes}\left(t_{1}, t_{2}\right), t_{3}\right), E_{\oplus}\left(E_{\oplus}\left(d_{1}, d_{2}\right), d_{3}\right)\right) \\
& =\left(E_{\otimes}\left(t_{1}, E_{\otimes}\left(t_{2}, t_{3}\right)\right), E_{\oplus}\left(d_{1}, E_{\oplus}\left(d_{2}, d_{3}\right)\right)\right)=P_{D}\left[\left(t_{1}, d_{1}\right),\left(E_{\otimes}\left(t_{2}, t_{3}\right), E_{\oplus}\left(d_{2}, d_{3}\right)\right)\right] \\
& =P_{D}\left[\lambda_{1}, P_{D}\left(\lambda_{2}, \lambda_{3}\right)\right]
\end{aligned}
$$

3. Monotonicity Criteria for $P_{D}$. Because $E_{\oplus}$ and $E_{\otimes}$ are monotonic, we have that $P_{D}$ does not decrease (increase) the trust [distrust] value when the chain trust [distrust] values increase (decrease).

4. Boundary Conditions for $P_{D}$. we have:

Full trust propagation: When $\lambda_{1}=(1,0)$ we have:

$$
P_{D}\left((1,0), \lambda_{2}\right)=\left(E_{\otimes}\left(1, t_{2}\right), E_{\oplus}\left(0, d_{2}\right)\right)=\left(t_{2}, d_{2}\right)=\lambda_{2}
$$

When $\lambda_{2}=(1,0)$, commutativity property implies that

$$
P_{D}\left(\lambda_{1},(1,0)\right)=P_{D}\left((1,0), \lambda_{1}\right)=\lambda_{1} .
$$


Thus, in a chain of three experts, if one expert fully trusts another expert, the TF of first expert to the third expert in the chain will be equal to the TF between the two other experts.

Full distrust propagation: When $\lambda_{1}=(0,1)$ we have:

$$
P_{D}\left((0,1), \lambda_{2}\right)=\left(E_{\otimes}\left(0, t_{2}\right), E_{\oplus}\left(1, d_{2}\right)\right)=(0,1)
$$

When $\lambda_{2}=(0,1)$, commutativity property implies that

$$
P_{D}\left(\lambda_{1},(0,1)\right)=P_{D}\left((0,1), \lambda_{1}\right)=(0,1) .
$$

Thus, in a chain of three experts, if one expert fully distrusts another expert, then the first expert will fully distrust the third expert in the chain no matter what the TF between the other two expert is.

Boundary consistency. In a chain of three experts in which the first expert fully trusts the second expert and this second expert fully distrusts the third expert, both above boundary conditions result in the same fully distrusts $\mathrm{TF}$ output, and therefore they are consistent with each other.

Example 1. Assume the following two TFs $\lambda_{1}=(0.8,0.4)$ and $\lambda_{2}=(0.7,0.5)$. Applying the dual trust propagation operator in expression (9), we obtain

$$
P_{D}\left(\lambda_{1}, \lambda_{2}\right)=(0.53,0.75)
$$

Using the propagating operator proposed by Victor et al. [29], we obtain

$$
P_{V}\left(\lambda_{1}, \lambda_{2}\right)=\left(t_{1} t_{2}, t_{1} d_{2}\right)=(0.56,0.4)
$$

Victor et al.'s trust propagating process makes the distrust value to decrease, which is not what is expected in real situations. Another limitation is that it does not take into account the distrust value in $\lambda_{1}$, and therefore monotonicity is not satisfied under Victor et al.'s trust propagating process. Indeed, if one of the distrust value is modified and $\lambda_{1}=(0.8,0.3)$ and $\lambda_{2}=(0.7,0.5)$, we obtain that:

$$
P_{D}\left(\lambda_{1}, \lambda_{2}\right)=(0.53,0.70)
$$

and

$$
P_{V}\left(\lambda_{1}, \lambda_{2}\right)=\left(t_{1} t_{2}, t_{1} d_{2}\right)=(0.56,0.4)
$$

It is clear that the proposed dual trust propagating operator $P_{D}$ can reflect the change of the distrust value in $\lambda_{1}$ while $P_{V}$ cannot.

In real decision making, a trust propagating chain may involve more than three experts. For example, in Fig. 1 there are two indirect paths from $E_{1}$ to $E_{2}$ that involve one other expert $\left[E_{3}\right.$ and 
$E_{5}$, respectively]; but also one path that involves three other experts $\left[\left(E_{3}, E_{4}, E_{5}\right)\right]$. In this last case, associativity property of $P_{D}$ can be applied and the following is obtained:

$$
P_{D}\left(\left(t_{1}, d_{1}\right),\left(t_{2}, d_{2}\right), \ldots,\left(t_{n}, d_{n}\right)\right)=\left(E_{\otimes}\left(t_{1}, t_{2}, \ldots, t_{n}\right), E_{\oplus}\left(d_{1}, d_{2}, \ldots, d_{n}\right)\right)
$$

Expressions (7) and (8) yield:

$$
P_{D}\left(\left(t_{1}, d_{1}\right),\left(t_{2}, d_{2}\right), \ldots,\left(t_{n}, d_{n}\right)\right)=\left(\frac{2 \prod_{i=1}^{n} t_{i}}{\prod_{i=1}^{n}\left(2-t_{i}\right)+\prod_{i=1}^{n} t_{i}}, \frac{\prod_{i=1}^{n}\left(1+d_{i}\right)-\prod_{i=1}^{n}\left(1-d_{i}\right)}{\prod_{i=1}^{n}\left(1+d_{i}\right)+\prod_{i=1}^{n}\left(1-d_{i}\right)}\right)
$$

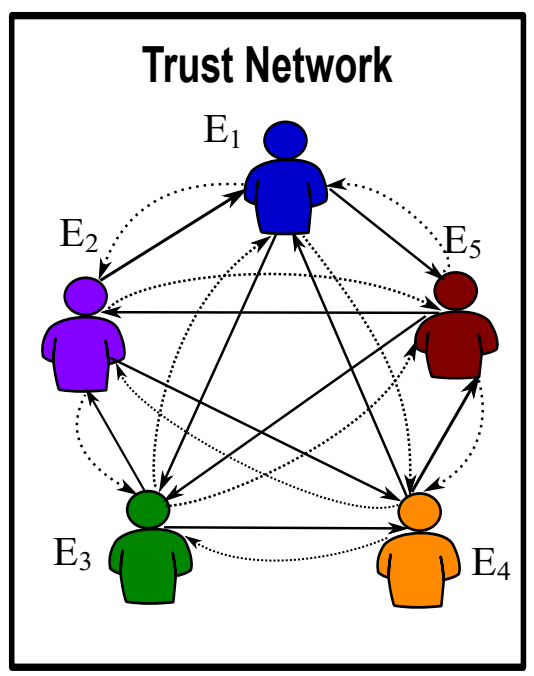

Figure 3: Fully Connected Trust Network after propagation: dash arrows means absence of direct trust computed via indirect TTPs

Example 2. A company invites a group of experts $\left(e_{1}, e_{2}, e_{3}, e_{4}, e_{5}\right)$ from five different departments to select the most appropriate cloud service supplier. They have the following a priori trust relationship as depicted in Figure 1, and corresponding incomplete trust sociomatrix $T_{L}$ :

$$
T_{L}=\left(\begin{array}{ccccc}
- & - & (0.6,0.1) & - & (0.8,0.1) \\
(0.8,0.5) & - & - & (0.7,0.5) & - \\
- & (0.7,0.2) & - & (0.5,0.2) & - \\
(0.8,0.3) & - & - & - & (0.6,0.3) \\
- & (0.8,0.5) & (0.3,0.1) & - & -
\end{array}\right)
$$

To fully connect the network with trust links between all the nodes as Figure 3 illustrates, indirect TTPs (Fig. 2(a)) to propagate TFs via dual trust propagation operation $P_{D}$ is used (Fig. 2(b)). For example, to propagate TFs from expert $E_{1}$ to expert $E_{2}$, there are five possible indirect TTPs paths: $L_{1}: E_{1} \rightarrow E_{3} \rightarrow E_{2} ; L_{2}: E_{1} \rightarrow E_{5} \rightarrow E_{2}$; and $L_{3}: E_{1} \rightarrow E_{3} \rightarrow E_{4} \rightarrow E_{5} \rightarrow E_{2}$. To reduce attenuation of information, only shortest indirect paths are used, and when more than one the average of the propagated TFs is computed. In this case, $L_{1}$ and $L_{2}$ are used to propagate TFs from $E_{1}$ to $E_{2}$. The computation involved is:

$$
P_{D}^{L_{1}}((0.6,0.1),(0.7,0.2))=(0.375,0.294)
$$




$$
\begin{gathered}
P_{D}^{L_{2}}((0.8,0.1),(0.8,0.5))=(0.615,0.574) \\
P_{D}^{E_{1} \rightarrow E_{2}}=\frac{P_{r}^{L_{1}}+P_{r}^{L_{2}}}{2}=(0.5,0.43)
\end{gathered}
$$

The completed trust sociomatrix would be:

$$
T_{L}=\left(\begin{array}{ccccc}
- & (0.50,0.43) & (0.60,0.10) & (0.25,0.29) & (0.80,0.10) \\
(0.80,0.50) & - & (0.44,0.57) & (0.70,0.50) & (0.50,0.63) \\
(0.53,0.64) & (0.70,0.20) & - & (0.50,0.20) & (0.25,0.47) \\
(0.80,0.30) & (0.44,0.70) & (0.29,0.39) & - & (0.60,0.30) \\
(0.62,0.80) & (0.80,0.50) & (0.30,0.10) & (0.32,0.55) & -
\end{array}\right)
$$

\section{Trust Based Aggregation of Decision Matrices}

\subsection{Trust Score of Experts}

When the complete trust relationship is constructed, each expert can be associated with a TS as follows [38]:

Definition 6. Let $G=(E, L, \omega)$ be a directed graph, $E=\left\{e_{1}, \ldots, e_{k}\right\}$ be the set of nodes and $L=\left\{l_{1}, \ldots, l_{q}\right\}$ be the set of directed lines, or arcs, between pairs of nodes and $S_{L}=\left(S_{l h}\right)_{k \times k}$ be the trust sociomatrix associated with the graph $G=(E, L, \omega)$, where $S_{l h}$ is the trust function from expert $E_{l}$ to expert $E_{h}$. Then

- The TF of expert $E_{h}$ is:

$$
\left(t_{h}, d_{h}\right)=\frac{1}{k-1} \sum_{l=1}^{k} S_{l h}
$$

- The TS of expert $E_{h}$ is :

$$
T S_{h}=\frac{t_{h}-d_{h}+1}{2}
$$

\subsection{Importance Weights by Trust Score}

TSs values can be used to assign importance weights to experts. Using Yager's OWA based procedure, experts importance weight are computed as follows [45, 46]:

$$
w_{T}^{\sigma(h)}=Q\left(\frac{T(\sigma(h))}{T(\sigma(k))}\right)-Q\left(\frac{T(\sigma(h-1))}{T(\sigma(k))}\right)
$$

with $T(\sigma(h))=\sum_{l=1}^{h} T S_{\sigma(l)}, \sigma$ is a permutation such that $T S_{\sigma(l)}$ is the $l$-th largest value of set $\left\{T S_{1}, \ldots, T S_{k}\right\}$ and $Q$ is a Basic Unit-interval Monotone (BUM) membership function of the fuzzy linguistic quantifier to implement in the aggregation process: $Q:[0,1] \rightarrow[0,1]$ such that $Q(0)=0$, $Q(1)=1$ and if $x>y$ then $Q(x) \geq Q(y)$. 


\subsection{Trust Based Collective Decision Matrix}

The individual decision matrices are aggregated into a collected one using the above TS based weights as follows:

Definition 7. Let $\left\{R^{(h)}=\left(r_{i j}^{(h)}\right)_{m \times n} ; h=1,2, \ldots, k\right\}$ be a collection of decision matrices given by a set of experts $E=\left\{e_{1}, \ldots, e_{k}\right\}$ with associated TSc $\left\{T S_{1}, \ldots, T S_{k}\right\}$. The collective decision matrix guided by trust is $\bar{R}=\left(\bar{r}_{i j}\right)_{m \times n}$ with element

$$
\bar{r}_{i j}=\sum_{h=1}^{k} w_{T}^{h} \cdot r_{i j}^{(h)}, i=1, \ldots, m ; j=1, \ldots, n
$$

where $w^{h}$ given by expression (14).

\section{Visual Interaction Method for Group Consensus}

Once the trust based collective decision matrix is obtained, consensus indexes (CI) at three levels are computed for each expert: (1) elements level; (2) alternatives level; and (3) decision matrix level. When the decision matrix level CI (group CI) reaches a set threshold value for all experts, the resolution process of the SN-GDM is carried out; otherwise the inconsistent experts are identified and invited to revisit some of their opinions that contribute less to consensus in order to increase the group CI. This paper proposes to do this by using a visual interaction method to provide expert with a visual representations of his/her consensus status. Additionally, a trust based recommendation mechanism is also developed to advice inconsistent experts, i.e experts with a lower consensus contribution than the set threshold value, on how to change their identified evaluation contributing less to consensus. This is complemented with a visual adoption mechanism to allow experts to 'see' their future consensus position if the recommendation advices were to be implemented. Using this visual extra information, the inconsistent experts can achieve a balance between group consensus and individual independence.

The visual interaction for SN-GDM with trust relationship is depicted in Figure 4. Specifically, it consists of the following five steps:

(1) Developing the dual trust propagation operator;

(2) Computing TS of experts and trust collective decision matrix;

(3) Determining CI at three levels;

(4) Visual consensus identification, trust based recommendation and visual adoption mechanism; and

(5) Selection Process.

The first and second steps have already been covered in Section 2 and Section 3, respectively. The remaining steps will be presented in more detail in the following subsections. 


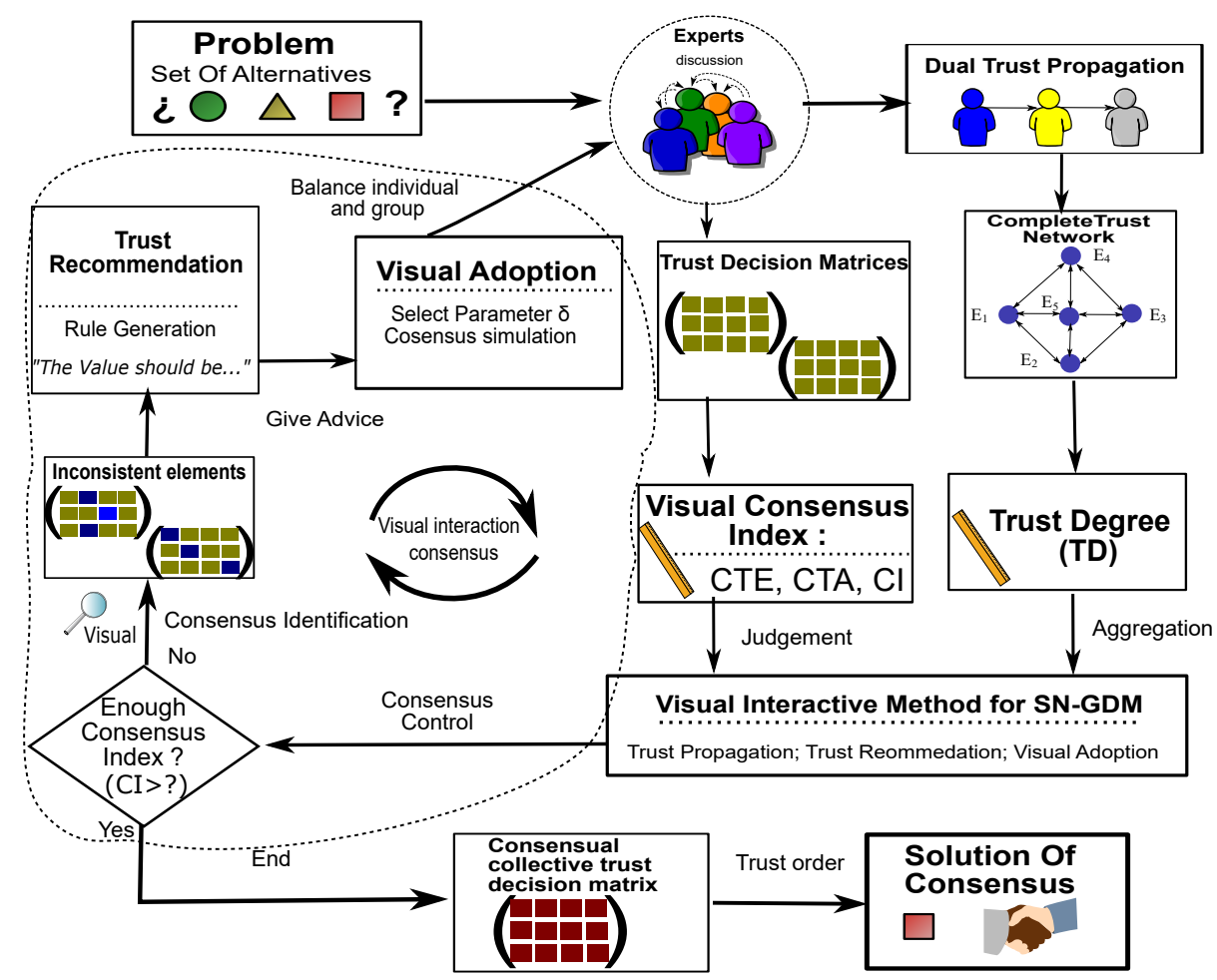

Figure 4: Visual interaction consensus model for SN-GDM with trust propagation

\subsection{Visual Identification of Three Consensus Levels}

The visual consensus model consists of three consecutive stages: (1) visual identification of the preference values with a consensus level lower than the set threshold value; (2) recommendation simulation for generating advice on the direction-value of the required change to increase current consensus; (3) visual recommendation process simulation to show what would happen if experts are to accept the recommended preference values.

First, the consensus index for each expert at the three different levels of a relation are computed as follow:

Level 1. Consensus index at element levels (pair of alternative-criterion). The consensus index of an expert $e_{h}$ with respect to the group on the alternatives $x_{i}$ under criterion $c_{j}$ is

$$
C T E_{i j}^{h}=1-d\left(r_{i j}^{h}, \bar{r}_{i j}\right)=1-\frac{\left|t_{i j}^{h}-\bar{t}_{i j}\right|+\left|d_{i j}^{h}-\bar{d}_{i j}\right|}{2}
$$

Level 2. Consensus index at alternatives level. The consensus index of an expert $e_{h}$ with respect to the group on the alternative $x_{i}$ is

$$
C T A_{i}^{h}=\frac{1}{n} \sum_{j=1}^{n} C T E_{i j}^{h}
$$


Level 3. Consensus index at decision matrix level. The consensus index of an expert $e_{h}$ to the group on decision matrix is

$$
C I^{h}=\frac{1}{m} \sum_{i=1}^{m} C T A_{i}^{h}
$$

The greater the value of $C I^{h}\left(0 \leq C I^{h} \leq 1\right)$, the greater the consensus index between individual expert $e_{h}$ and the group. When $C I^{h}=1$, this means that expert $e_{h}$ has same decision matrix as the group collective decision matrix (unanimous agreement). However, this case is rare in practice and possibly impractical nor desirable in a decision making context. Consequently, a consensus threshold $\gamma$ such as $90 \%, 80 \%$, two - thirds are commonly set as a minimum level to achieve [26]. When at least a $C I^{h}$ is less than the predefined threshold $\gamma$, a visual interaction procedure is activated to help inconsistent experts see where they are and what can be changed to increase their consensus with respect to the group. Otherwise, an appropriate selection process is applied to derive the solution of consensus.

The visual identification uses visual graphs to help experts 'see' their consensus position within the group as illustrated in Figure 5. Using the threshold value $\gamma=0.9$, Figure 5(a) presents a visual representation of all experts consensus level with respect to the threshold value. It is clear that $e_{4}$ is identified as the only expert contributing less to group consensus. Additionally, individual visual representations of consensus indexes at alternatives and elements levels, respectively, are also provided to the identified experts at the decision matrix level to help them see in which alternatives and in which preference values he/she is contributing less to consensus. Mathematically, these steps are modelled as follows:
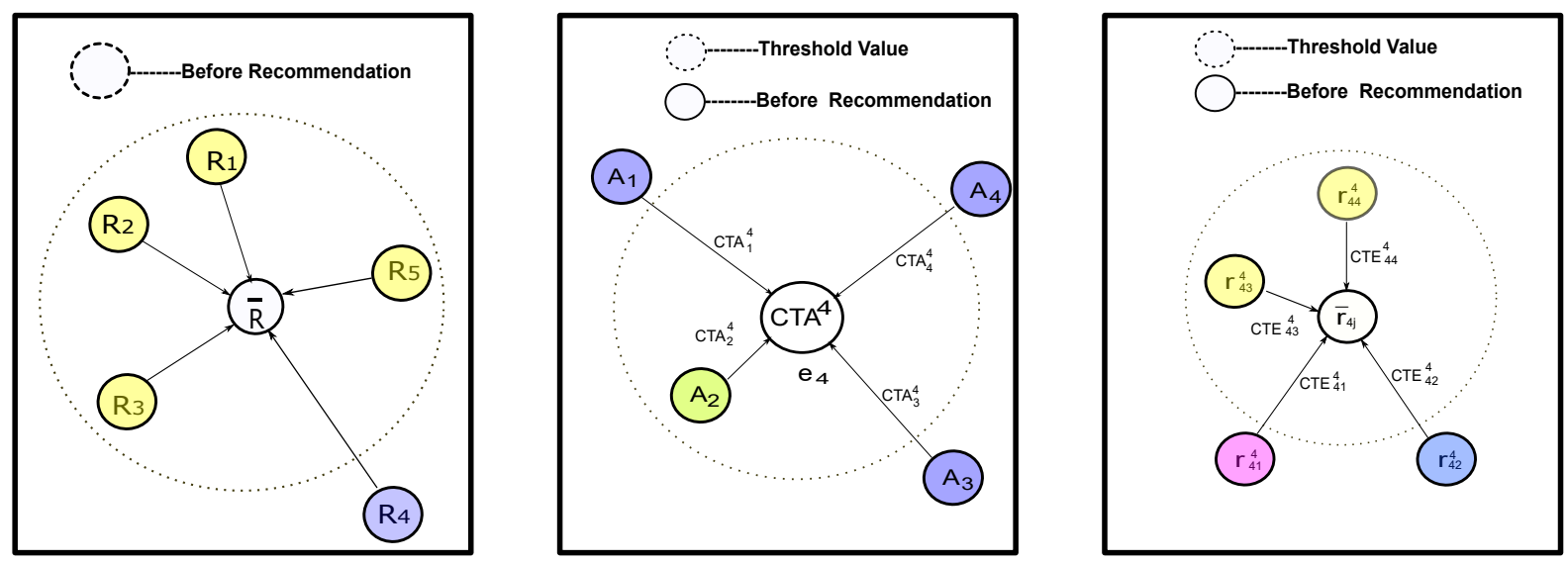

(a) The visual consensus index at the (b) The visual consensus index at the (c) The visual consensus index at the decision matrix level: alternatives level for expert $e_{4}$ element $A_{4}$ level for expert $e_{4}$

Figure 5: Three visual levels of consensus before adopting the recommendation advice

Step 1. Experts with a consensus index at decision matrix lower than the threshold value $\gamma$ are 
identified:

$$
E X P C H=\left\{h \mid C I^{h}<\gamma\right\}
$$

Step 2. For the identified experts in Step 1, their alternatives with a consensus index $C T A_{i}^{h}$ lower than the threshold $\gamma$ are identified:

$$
A L T=\left\{(h, i) \mid h \in E X P C H \wedge C T A_{i}^{h}<\gamma\right\}
$$

Step 3. Finally, the preference values to be changed are those with a consensus index $C T E_{i}^{h}$ lower than the threshold $\gamma$ :

$$
A P S=\left\{(h, i, j) \mid(h, i) \in A L T \wedge C T E_{i j}^{h}<\gamma\right\}
$$

\subsection{Trust based Recommendation for Generating Advice}

The recommendation produces personalised advice for experts on how to modify their assessment values to increase their consensus level. Given $(h, i, j) \in A P S$, expert $e_{h}$ receives the following personalised advice rules:

1. "You should change your evaluation for alternative $x_{i}$ under criteria $c_{j}, r_{i j}^{h}$, to a value closer to $r r_{i j}^{h} . "$

$$
r r_{i j}^{h}=(1-\delta) \cdot r_{i j}^{h}+\delta \cdot \bar{r}_{i j}
$$

where $\delta \in[0,1]$ is a parameter to control the degree of advice and $\bar{r}_{i j}$ is the trust based collective evaluation as per Definition 7.

\subsection{Visual Adoption Mechanism for Consensus}

Notice that when the feedback parameter $\delta$ takes value 1 , the original assessment is completely replaced by the trust based collective evaluation using the above recommendation rule, while when the feedback parameter $\delta$ takes value 0 the original assessment is kept unchanged. It would be interesting to know as well how the consensus index at the elements level $C T E_{i j}^{h}$ is affected by the feedback parameter $\delta$ when changes are implemented, so that experts can analyse which $\delta$ value is best to achieve an acceptable compromise between group consensus and individual independence.

Notice that the new recommended values are closer than the original values to the collective evaluation, which when fixed will imply that the consensus index at elements level would increase with the value of the parameter $\delta$. An extreme case of this scenario would derive with the production of recommendations to all experts on decision elements for all pairs of alternative-criterion. The implementation of the recommended values will guarantee that all expert consensus index at the elements values will increase values increase. This is proved in the following: 
Lemma 1. Let $\left\{R^{h}=\left(r_{i j}^{h}\right)_{m \times n} ; h=1,2, \ldots, k\right\}$ be a collection of decision matrices given by a set of experts $E=\left\{e_{1}, \ldots, e_{k}\right\}$ with associated TSc $\left\{T S_{1}, \ldots, T S_{k}\right\}$. Let $\bar{R}=\left(\bar{r}_{i j}\right)_{m \times n}$ be the collective decision matrix guided by trust. Let $\left\{r R^{h}=\left(r r_{i j}^{h}\right)_{m \times n} ; h=1,2, \ldots, k\right\}$ be the set of new decision matrices with

$$
r r_{i j}^{h}=(1-\delta) \cdot r_{i j}^{h}+\delta \cdot \bar{r}_{i j} ; \delta \in[0,1]
$$

and $\overline{r R}$ be their corresponding collective decision matrix guided by trust. Then, it is:

$$
\overline{r R}=\bar{R}
$$

Proof. Expression (14) is used to derive weighting vector $\left\{w_{T}^{h} ; h=1,2, \ldots, k\right\}$ to compute the collective decision matrices as per Definition 7:

$$
\begin{aligned}
\overline{r r}_{i j} & =\sum_{h=1}^{k} w_{T}^{h} \cdot r r_{i j}^{h}=\sum_{h=1}^{k} w_{T}^{h} \cdot\left[(1-\delta) \cdot r_{i j}^{h}+\delta \cdot \bar{r}_{i j}\right] \\
& =(1-\delta) \cdot \sum_{h=1}^{k} w_{T}^{h} \cdot r_{i j}^{h}+\delta \cdot \bar{r}_{i j} \cdot \sum_{h=1}^{k} w_{T}^{h}=(1-\delta) \cdot \bar{r}_{i j}+\delta \cdot \bar{r}_{i j} \\
& =\bar{r}_{i j}
\end{aligned}
$$

Proposition 1. Under the condition of Lemma 1 we have: $d\left(r r_{i j}^{h}, \overline{r r}_{i j}\right) \leq d\left(r_{i j}^{h}, \bar{r}_{i j}\right)$.

Proof. Because $d\left(r_{i j}^{h}, \bar{r}_{i j}\right)=\frac{\left|t_{i j}^{h}-\bar{t}_{i j}\right|+\left|d_{i j}^{h}-\bar{d}_{i j}\right|}{2}$, to prove that $d\left(r r_{i j}^{h}, \overline{r r}_{i j}\right) \leq d\left(r_{i j}^{h}, \bar{r}_{i j}\right)$ we only need to prove that $d\left(r t_{i j}^{h}, \overline{r t}_{i j}\right) \leq d\left(t_{i j}^{h}, \bar{t}_{i j}\right)$ and $d\left(r d_{i j}^{h}, \overline{r d}_{i j}\right) \leq d\left(d_{i j}^{h}, \bar{d}_{i j}\right)$. Applying Lemma 1, we have:

$$
d\left(r t_{i j}^{h}, \overline{r t}_{i j}\right)=\left|r t_{i j}^{h}-\overline{r t}_{i j}\right|=\left|(1-\delta) \cdot t_{i j}^{h}+\delta \cdot \bar{t}_{i j}-\bar{t}_{i j}\right|=(1-\delta) \cdot\left|t_{i j}^{h}-\bar{t}_{i j}\right| \leq\left|t_{i j}^{h}-\bar{t}_{i j}\right|=d\left(t_{i j}^{h}, \bar{t}_{i j}\right)
$$

Similarly, we can prove that $d\left(r d_{i j}^{h}, \overline{r d}_{i j}\right) \leq d\left(d_{i j}^{h}, \bar{d}_{i j}\right)$.

Because $d\left(r r_{i j}^{h}, \overline{r r}_{i j}\right)=(1-\delta) \cdot d\left(r_{i j}^{h}, \bar{r}_{i j}\right)$, we have that the feedback mechanism would make the consensus process to converge to a unanimous consensus when $\delta>0$. As mentioned before, the above results are only valid when all experts change all their assessment values to the recommendation ones. Therefore, the production of recommendations to all experts and their implementation will guarantee that experts consensus index at elements level will increase, and consequently the group consensus index will also increase (monotonically with respect to the parameter $\delta$ ). However, in real applications all experts will not receive recommendations to change values at alternative level, otherwise the computational complexity of the decision making process would increase. Therefore, in the consensus process proposed here only those experts with a consensus level lower than the threshold value will receive recommendations, and the previous results are not applicable. Indeed, the collective decision matrix when changes are implemented by just some experts on some of their values is not guaranteed to be the same than before the implementation of changes. It is expected though that 
those experts receiving recommendations and implementing recommended values will become closer to the new collective decision matrix than before the implementation of changes, and in turn it would lead to an increase of their consensus index at elements level. Obviously, the same conclusion cannot be drawn for the experts that do not change their original assessment values. The following result indicates that if advices are implemented by just one expert, then this expert's consensus index at elements level will increase.

Proposition 2. If only expert $e_{s}$ adopts the recommended advices then the new consensus index at element level for such expert will be greater than or equal to his/her previous consensus index at element level. Furthermore, the new consensus index at element level is monotonic increasing with respect to parameter $\delta$.

Proof. After the expert $e_{s}$ adopts recommended value for alternative $x_{i}$ under criteria $c_{j}$, then assessment values for alternative $x_{i}$ under criteria $c_{j}$ are divided into two groups: the new preference value $\left\{r r_{i j}^{s} \mid r r_{i j}^{s}=(1-\delta) r_{i j}^{h}+\delta \times \bar{r}_{i j}\right\}$ and the set of unchanged assessment values $\left\{\left(r r_{i j}^{h}\right) \mid r r_{i j}^{h}=r_{i j}^{h}, h=\right.$ $1, \cdots, k, h \neq s\}$. Then, we have

$$
\overline{r r}_{i j}=w_{T}^{s} \cdot r r_{i j}^{s}+\sum_{h=1, h \neq s}^{k} w_{T}^{h} \cdot r r_{i j}^{h}=w_{T}^{s} \cdot r r_{i j}^{s}-w_{T}^{s} \cdot r_{i j}^{s}+\bar{r}_{i j}
$$

and the new consensus index for alternative $x_{i}$ under criteria $c_{j}$, which we will denote by $\overline{C T E}_{i j}$ to differentiate it from the previous one $C T E_{i j}$, would be

$$
\begin{aligned}
\overline{C T E}_{i j}^{s} & =1-\left|r r_{i j}^{s}-\overline{r r}_{i j}\right| \\
& =1-\left|(1-\delta) \cdot r_{i j}^{s}+\delta \cdot \bar{r}_{i j}-\left(w_{T}^{s} \cdot r r_{i j}^{s}-w_{T}^{s} \cdot r_{i j}^{s}+\bar{r}_{i j}\right)\right| \\
& =1-\left|(1-\delta) \cdot r_{i j}^{s}+\delta \cdot \bar{r}_{i j}-\left(w_{T}^{s} \cdot\left((1-\delta) \cdot r_{i j}^{s}+\delta \bar{r}_{i j}\right)-w_{T}^{s} \cdot r_{i j}^{s}+\bar{r}_{i j}\right)\right| \\
& =1-\left[1-\delta \cdot\left(1-w_{T}^{s}\right)\right] \cdot\left|r_{i j}^{s}-\bar{r}_{i j}\right|
\end{aligned}
$$

Because $w_{T}^{s}, \delta \in[0,1]$, it is $1-\delta \leq 1-\delta \cdot\left(1-w_{T}^{s}\right) \leq 1$, and consequently we will have that the new consensus index for alternative $x_{i}$ under criteria $c_{j}$ for expert $e_{s}$ will be greater than or equal to the previous consensus index for alternative $x_{i}$ under criteria before implementing the corresponding advice valued. The increment of the new consensus index with respect to the previous consensus index for alternative $x_{i}$ under criteria $c_{j}$ for expert $e_{s}$ will be:

$$
\overline{C T E}_{i j}^{h}-C T E_{i j}^{h}=\delta \cdot\left(1-w_{T}^{s}\right) \cdot\left|r_{i j}^{s}-\bar{r}_{i j}\right| \cdot
$$

Equality between the new consensus index and the previous one happens only when parameter $\delta=0$, i.e when no changes are made to the original assessment for alternative $x_{i}$ under criteria $c_{j}$. Obviously, the consensus index does not change if original assessment is not changed. In conclusion, experts $e_{s}$ new consensus index at element level is monotonic increasing with respect to parameter $\delta$ when recommended advices values are implemented. 
Proposition 2 can be used to implement a policy of implementation of recommended advices with one expert at a time starting with the most inconsistent one. The most common policy as explained before is to recommend advices on changes of assessment at elements level to all those experts with a consistency index below the threshold value. When this is the case, for those experts that implement recommended advice values, $e_{s}$, we can obtain the range of the difference between their new consensus index with respect to their previous consensus index for alternative $x_{i}$ under criteria $c_{j}$. This is presented in the following:

Proposition 3. The difference in absolute value between the new consensus index with respect to their previous consensus index for alternative $x_{i}$ under criteria $c_{j}$ after inconsistent experts implement recommended changes are:

- For inconsistent experts: $\left|\overline{C T E}_{i j}^{s}-C T E_{i j}^{s}\right| \leq \delta \cdot\left[\left|\sum_{h=1}^{l} w_{T}^{h} \cdot\left(r_{i j}^{h}-\bar{r}_{i j}\right)\right|+1\right]$

- For consistent experts: $\left|\overline{C T E}_{i j}^{q}-C T E_{i j}^{q}\right| \leq \delta \cdot\left|\sum_{h=1}^{l} w_{T}^{h} \cdot\left(r_{i j}^{h}-\bar{r}_{i j}\right)\right|$

Proof. After the experts implement recommendation advice, the assessment values for alternative $x_{i}$ under criteria $c_{j}$ can be divided into two groups: the set of new assessment values $\left\{\left(r r_{i j}^{s}\right) \mid r r_{i j}^{s}=\right.$ $\left.(1-\delta) \cdot r_{i j}^{s}+\delta \cdot \bar{r}_{i j} ; s=1, \cdots, l\right\}$ implemented by inconsistent experts, and the set of original assessment values $\left\{\left(r r_{i j}^{h}\right) \mid r_{i j}^{q}=r_{i j}^{q} ; q=l+1, \cdots, k\right\}$ corresponding to the consistent experts. Using these two sets of assessment values, we have:

$$
\begin{aligned}
\overline{r r}_{i j} & =\sum_{h=1}^{l} w_{T}^{h} \cdot r r_{i j}^{h}+\sum_{h=l+1}^{k} w_{T}^{h} \cdot r_{i j}^{h} \\
& =\sum_{h=1}^{l} w_{T}^{h} \cdot\left[(1-\delta) \cdot r_{i j}^{h}+\delta \cdot \bar{r}_{i j}\right]+\sum_{h=l+1}^{k} w_{T}^{h} \cdot r_{i j}^{h} \\
& =\bar{r}_{i j}-\sum_{h=1}^{l} w_{T}^{h} \cdot \delta \cdot\left(r_{i j}^{h}-\bar{r}_{i j}\right)
\end{aligned}
$$

- For $s \in\{1, \cdots, l\}$,it is:

$$
\begin{aligned}
\overline{C T E}_{i j}^{s} & =1-d\left(r r_{i j}^{s}-\overline{r r}_{i j}\right) \\
& =1-\left|\left[(1-\delta) \cdot r_{i j}^{s}+\delta \cdot \bar{r}_{i j}\right]-\left[\bar{r}_{i j}-\sum_{h=1}^{l} w_{T}^{h} \cdot \delta \cdot\left(r_{i j}^{h}-\bar{r}_{i j}\right)\right]\right| \\
& =1-\left|(1-\delta) \cdot\left(r_{i j}^{s}-\bar{r}_{i j}\right)+\sum_{h=1}^{l} w_{T}^{h} \cdot \delta \cdot\left(r_{i j}^{h}-\bar{r}_{i j}\right)\right|
\end{aligned}
$$


- Applying that $|a+b| \geq|a|-|b|$ we have

$$
\begin{aligned}
\overline{C T E}_{i j}^{s} & \leq 1-(1-\delta) \cdot\left|r_{i j}^{s}-\bar{r}_{i j}\right|+\delta \cdot\left|\sum_{h=1}^{l} w_{T}^{h} \cdot\left(r_{i j}^{h}-\bar{r}_{i j}\right)\right| \\
& =\delta+(1-\delta)-(1-\delta) \cdot\left|r_{i j}^{s}-\bar{r}_{i j}\right|+\delta \cdot\left|\sum_{h=1}^{l} w_{T}^{h} \cdot\left(r_{i j}^{h}-\bar{r}_{i j}\right)\right| \\
& =\delta+(1-\delta) \cdot\left[1-\left|r_{i j}^{s}-\bar{r}_{i j}\right|\right]+\delta \cdot\left|\sum_{h=1}^{l} w_{T}^{h} \cdot\left(r_{i j}^{h}-\bar{r}_{i j}\right)\right| \\
& \leq 1-d\left(r_{i j}^{s}, \bar{r}_{i j}\right)+\delta \cdot\left[\left|\sum_{h=1}^{l} w_{T}^{h} \cdot\left(r_{i j}^{h}-\bar{r}_{i j}\right)\right|+1\right]
\end{aligned}
$$

Thus,

$$
\overline{C T E}_{i j}^{s}-C T E_{i j}^{s} \leq \delta \cdot\left[\left|\sum_{h=1}^{l} w_{T}^{h} \cdot\left(r_{i j}^{h}-\bar{r}_{i j}\right)\right|+1\right]
$$

- Applying that $|a+b| \leq|a|+|b|$ we have

$$
\begin{aligned}
\overline{C T E}_{i j}^{s} & \geq 1-(1-\delta) \cdot\left|r_{i j}^{s}-\bar{r}_{i j}\right|-\delta \cdot\left|\sum_{h=1}^{l} w_{T}^{h} \cdot\left(r_{i j}^{h}-\bar{r}_{i j}\right)\right| \\
& \geq 1-\left|r_{i j}^{s}-\bar{r}_{i j}\right|-\delta \cdot\left|\sum_{h=1}^{l} w_{T}^{h} \cdot\left(r_{i j}^{h}-\bar{r}_{i j}\right)\right| \\
& \geq 1-d\left(r_{i j}^{s}, \bar{r}_{i j}\right)-\delta \cdot\left[\left|\sum_{h=1}^{l} w_{T}^{h} \cdot\left(r_{i j}^{h}-\bar{r}_{i j}\right)\right|+1\right]
\end{aligned}
$$

Thus,

$$
-\delta \cdot\left[\left|\sum_{h=1}^{l} w_{T}^{h} \cdot\left(r_{i j}^{h}-\bar{r}_{i j}\right)\right|+1\right] \leq \overline{C T E}_{i j}^{s}-C T E_{i j}^{s}
$$

We conclude that when $s \in\{1, \cdots, l\}$ it is:

$$
\left|\overline{C T E}_{i j}^{s}-C T E_{i j}^{s}\right| \leq \delta \cdot\left[\left|\sum_{h=1}^{l} w_{T}^{h} \cdot\left(r_{i j}^{h}-\bar{r}_{i j}\right)\right|+1\right]
$$

- For $q \in\{l+1, \cdots, k\}$ it is:

$$
\begin{aligned}
\overline{C T E}_{i j}^{q} & =1-d\left(r r_{i j}^{q}-\overline{r r}_{i j}\right) \\
& =1-\left|r_{i j}^{q}-\left[\bar{r}_{i j}-\sum_{h=1}^{l} w_{T}^{h} \cdot \delta \cdot\left(r_{i j}^{h}-\bar{r}_{i j}\right)\right]\right| \\
& =1-\left|\left(r_{i j}^{q}-\bar{r}_{i j}\right)+\sum_{h=1}^{l} w_{T}^{h} \cdot \delta \cdot\left(r_{i j}^{h}-\bar{r}_{i j}\right)\right|
\end{aligned}
$$

Similarly to the previous case, we can conclude that when $q \in\{l+1, \cdots, k\}$ it is:

$$
\left|\overline{C T E}_{i j}^{q}-C T E_{i j}^{q}\right| \leq \delta \cdot\left|\sum_{h=1}^{l} w_{T}^{h} \cdot\left(r_{i j}^{h}-\bar{r}_{i j}\right)\right|
$$


Thus the range of the difference in absolute value between the consensus index at the alternatives level in subsequent steps following the implementation of the advice rules decreases when parameter $\delta$ decreases, being zero when $\delta=0$. However, we cannot guarantee that $\overline{C T E}_{i j}^{h}$ is bigger than $C T E_{i j}^{h}$. Also, we cannot guarantee that $\overline{C T E}_{i j}^{h}$ increases with respect to $\delta$.

Proposition 3 states clearly that the range of the difference in absolute value between the consensus degree at elements level when only inconsistent experts change their values is monotonic increasing with respect to the parameter $\delta$, and therefore there is much room of improvement of consensus the bigger $\delta$ is chosen. This would be used as evidence that high values of $\delta$ push inconsistent experts towards the group and therefore will increase the group consensus at the cost of the individual independence, which is kept the lower the parameter $\delta$ is selected. Thus, it is up to the experts to willingly propose a $\delta$ parameter that represents his/her own compromise and an acceptable balance for him/her between the group consensus and his/her own independence $[6,8,10,11]$. To support inconsistent experts in selecting the minimum $\delta$ value that place them as consistent experts in the following round, a visual adoption mechanism for recommendation advices with three steps is given below and illustrated in Fig 6.

Step 1. Choose different values for parameter $\delta$ from zero to one $\left(\delta_{i}\right)$, compute corresponding consensus indexes and visualise simulation of new consensus state (Fig 6(a)).

Step 2. Applying a what-if scenario analysis, the experts can easily compute and see their consensus status with respect to the rest of the group by choosing different parameters $\delta_{i}$ as per Table 1 , and then select the minimum value $\delta_{\min }$ that satisfies the consensus threshold criterion (Fig $(6(b))$.

Step 3. Using $\delta_{\min }$ and Expression (19), visual adoption mechanism for recommendation produces personalised advice for experts according to their own willingness.

\subsection{Selection Process}

Each alternative is associated a final weighted criteria collective assessments

$$
\bar{r}_{i}=\sum_{j=1}^{n} w_{j} \cdot \bar{r}_{i j}
$$

Their corresponding trust degrees can be used as final score values to produce a total ordering of alternatives from which to select the best alternative. 


\section{Framework of the visual interaction model for consensus in SN-GDM with trust prop- agation}

5.1. Description of the visual interaction model for consensus in SN-GDM with trust propagation problem and its components

The decision problem is how to achieve consensus in selecting the best alternative(s) from a set of feasible ones according to the evaluations provided by a group of experts socially networked with (possibly incomplete) trust statements (depicted in Figure 4). The following notations are used to denote the sets and variables used in the proposed visual interaction consensus model with trust propagation:

- $X=\left\{x_{1}, x_{2}, \ldots, x_{m}\right\}$ is the set of $n$ alternatives.

- $E=\left\{e_{1}, e_{2}, \ldots, e_{k}\right\}$ is the set of $k$ experts socially networked with (incomplete) trust values; $w_{h}$ is the importance degree of $h$-th expert, which is assumed to verify $\sum_{h=1}^{k} w_{h}=1, w_{h} \in[0,1]$, but it is unknown.

- $C=\left\{c_{1}, c_{2}, \ldots, c_{n}\right\}$ is the set of $m$ criteria for evaluating alternatives, where $\omega_{j}$ is the associated weight of criterion $c_{j}$, which verify $\sum_{j=1}^{n} \omega_{j}=1, \omega_{j} \in[0,1], j=1,2, \ldots, n$.

- $\left\{R^{(h)}=\left(r_{i j}^{(h)}\right)_{m \times n} ; h=1,2, \ldots, k\right\}$ is a collection of decision matrices given by the set of experts $E$, where $r_{i j}^{(h)}$ is the expert $e_{h}$ evaluation of how well alternative $x_{i}$ fulfil criterion $c_{j}$.

5.2. Decision process of the visual interaction model for consensus in SN-GDM with trust propagation

Step 1. Experts in the networked group express their original (possibly incomplete) trust opinions on others experts in the group, and (possibly incomplete/not fully connected) trust network is constructed (Figure 1). If required, trust propagation operator $P_{D}(10)$ is applied to establish the complete (fully connected) trust network (Figure 3).

Step 2. Trust scores of each expert are computed applying (13) and used to assign importance degrees to each expert applying (14). Individual decision matrices are aggregated into a collective one $\bar{R}=\left(\bar{r}_{i j}\right)_{m \times n}$ applying (15).

Step 3. Three different levels of consensus indexes for each expert are computed using (16), (18) and (17), respectively. Set a predefined threshold $\gamma$ to create a visual representation of all experts consensus level with respect to the threshold value and to identify experts contributing less to group consensus.

Step 4. Activate recommendation mechanism to produce advice (19) to experts identify in previous step. The visual adoption mechanism for determining the appropriate parameter $\delta$ (Fig 6) is provided. 
Step 5. Experts implement their new preference values following the provided recommendations and all the individual decision matrices are aggregated into a collective one $\overline{\bar{R}}$. If consensus degree threshold $\gamma$ is achieved, then proceed to next step, otherwise go to Step 3.

Step 6. Selection process is activated by aggregating the collective assessments of each alternative with a final weighted criteria using selection (20) and producing final ranking of alternatives using (4).

\section{Numerical example}

A company needs to select the most appropriate cloud service supplier from four possible alternatives: $\left\{x_{1}, x_{2}, x_{3}, x_{4}\right\}$. Four criteria $\left\{c_{1}, c_{2}, c_{3}, c_{4}, c_{5}\right\}$ are considered: Performance; Security; Privacy; Usability and Accessibility, with associated weighting vector $\omega=(0.25,0.40,0.15,0.20)^{T}$. A group of experts $\left\{e_{1}, e_{2}, e_{3}, e_{4}, e_{5}\right\}$ from five different departments with following priori trust relationship as depicted in Figure 1.

Step 1. With the original incomplete trust network (Figure 1), the complete trust network (Figure 3 ) is constructed using the trust propagation operator $P_{D}(10)$.

Step 2. According to expression (12), the following TFs of experts are obtained:

$$
\lambda_{1}=[0.69,0.56] ; \quad \lambda_{2}=[0.61,0.46] ; \quad \lambda_{3}=[0.41,0.29] ; \quad \lambda_{4}=[0.44,0.39] ; \quad \lambda_{5}=[0.54,0.38] .
$$

The TSs of expert are:

$$
T S_{1}=0.565 ; \quad T S_{2}=0.575 ; \quad T S_{3}=0.560 ; \quad T S_{4}=0.525 ; \quad T S_{5}=0.580
$$

It is:

$$
\lambda_{\sigma(1)}=\lambda_{5} ; \quad \lambda_{\sigma(2)}=\lambda_{2} ; \quad \lambda_{\sigma(3)}=\lambda_{1} ; \quad \lambda_{\sigma(4)}=\lambda_{3} ; \quad \lambda_{\sigma(5)}=\lambda_{4}
$$

Using the BUM function $Q(r)=r^{2 / 3}$, the following importance weights are derived:

$$
w_{T}^{\sigma(1)}=0.35 ; \quad w_{T}^{\sigma(2)}=0.20 ; \quad w_{T}^{\sigma(3)}=0.17 ; \quad w_{T}^{\sigma(4)}=0.15 ; \quad w_{T}^{\sigma(5)}=0.13
$$

The corresponding experts weights are:

$$
w_{T}^{1}=0.17 ; \quad w_{T}^{2}=0.20 ; \quad w_{T}^{3}=0.15 ; \quad w_{T}^{4}=0.13 ; \quad w_{T}^{5}=0.35
$$

Assume the five experts give the evaluation of the four cloud service suppliers via the following decision matrices:

$$
R^{1}=\left(\begin{array}{cccc}
(0.5,0.5) & (0.3,0.5) & (0.4,0.6) & (0.5,0.6) \\
(0.5,0.6) & (0.6,0.4) & (0.5,0.6) & (0.8,0.7) \\
(0.4,0.7) & (0.2,0.5) & (0.5,0.5) & (0.4,0.5) \\
(0.3,0.6) & (0.4,0.4) & (0.4,0.6) & (0.5,0.5)
\end{array}\right)
$$




$$
\begin{aligned}
R^{2} & =\left(\begin{array}{llll}
(0.5,0.5) & (0.4,0.5) & (0.3,0.6) & (0.4,0.6) \\
(0.5,0.7) & (0.5,0.5) & (0.5,0.8) & (0.6,0.6) \\
(0.6,0.4) & (0.4,0.5) & (0.5,0.5) & (0.4,0.6) \\
(0.4,0.5) & (0.3,0.5) & (0.5,0.6) & (0.5,0.5)
\end{array}\right) \\
R^{3} & =\left(\begin{array}{llll}
(0.5,0.5) & (0.3,0.6) & (0.4,0.6) & (0.4,0.6) \\
(0.4,0.6) & (0.5,0.5) & (0.3,0.5) & (0.4,0.7) \\
(0.5,0.5) & (0.5,0.6) & (0.5,0.5) & (0.6,0.5) \\
(0.7,0.5) & (0.6,0.5) & (0.3,0.6) & (0.5,0.5)
\end{array}\right) \\
R^{4} & =\left(\begin{array}{llll}
(0.6,0.5) & (0.7,0.6) & (0.5,0.5) & (0.6,0.1) \\
(0.4,0.6) & (0.5,0.5) & (0.6,0.6) & (0.5,0.4) \\
(0.2,0.6) & (0.5,0.7) & (0.7,0.3) & (0.8,0.7) \\
(0.2,0.8) & (0.3,0.7) & (0.5,0.4) & (0.4,0.6)
\end{array}\right) \\
R^{5} & =\left(\begin{array}{llll}
(0.4,0.2) & (0.4,0.6) & (0.6,0.8) & (0.5,0.2) \\
(0.5,0.8) & (0.4,0.2) & (0.5,0.3) & (0.4,0.7) \\
(0.3,0.4) & (0.4,0.6) & (0.6,0.8) & (0.7,0.3) \\
(0.4,0.6) & (0.7,0.4) & (0.5,0.6) & (0.6,0.7)
\end{array}\right)
\end{aligned}
$$

According to expression (15), the trust based collective decision matrix is calculated:

$$
\bar{R}=\left(\begin{array}{cccc}
(0.478,0.395) & (0.422,0.563) & (0.448,0.657) & (0.478,0.395) \\
(0.487,0.690) & (0.482,0.378) & (0.513,0.520) & (0.551,0.641) \\
(0.409,0.492) & (0.379,0.576) & (0.561,0.579) & (0.557,0.476) \\
(0.357,0.591) & (0.457,0.474) & (0.483,0.574) & (0.522,0.583)
\end{array}\right)
$$

Step 3. The consensus indexes at the pair of alternative-criterion level are:

$$
\begin{gathered}
C T E^{1}=\left(\begin{array}{llll}
0.937 & 0.908 & 0.948 & 0.887 \\
0.949 & 0.930 & 0.954 & 0.846 \\
0.892 & 0.873 & 0.930 & 0.910 \\
0.967 & 0.935 & 0.946 & 0.948
\end{array}\right) \quad C T E^{2}=\left(\begin{array}{lllll}
0.937 & 0.958 & 0.898 & 0.859 \\
0.989 & 0.930 & 0.854 & 0.956 \\
0.859 & 0.952 & 0.930 & 0.860 \\
0.933 & 0.909 & 0.979 & 0.948
\end{array}\right) \\
C T E^{3}=\left(\begin{array}{lllll}
0.937 & 0.921 & 0.948 & 0.859 \\
0.912 & 0.930 & 0.884 & 0.890 \\
0.951 & 0.928 & 0.9300 & 0.967 \\
0.783 & 0.916 & 0.895 & 0.948
\end{array}\right) \quad C T E^{4}=\left(\begin{array}{llllll}
0.887 & 0.843 & 0.896 & 0.792 \\
0.912 & 0.930 & 0.917 & 0.854 \\
0.842 & 0.878 & 0.791 & 0.767 \\
0.817 & 0.809 & 0.905 & 0.931
\end{array}\right) \\
C T E^{5}=\left(\begin{array}{lllll}
0.864 & 0.971 & 0.853 & 0.892 \\
0.938 & 0.870 & 0.884 & 0.895 \\
0.899 & 0.978 & 0.870 & 0.841 \\
0.974 & 0.842 & 0.979 & 0.921
\end{array}\right)
\end{gathered}
$$


The consensus indexes at the alternatives level are:

$$
\begin{aligned}
& C T A^{1}=(0.919,0.919,0.901,0.947) ; \quad C T A^{2}=(0.913,0.932,0.900,0.942) ; \\
& C T A^{3}=(0.916,0.905,0.944,0.885) ; \quad C T A^{4}=(0.854,0.903,0.819,0.865) ; \\
& C T A^{5}=(0.919,0.919,0.901,0.949) .
\end{aligned}
$$

The individual consensus indexes at the decision matrix level are:

$$
C I^{1}=0.922, C I^{2}=0.921, C I^{3}=0.912, C I^{4}=0.860, C I^{5}=0.903 .
$$

With a set threshold value of $\gamma=0.9$, the recommendation simulation is activated to assist expert $e_{4}$ to modify his/her some of original preference values to increase his/her consensus status. The following APS set is obtained:

$$
A P S=\{(4,1,1),(4,1,2),(4,1,3),(4,1,4),(4,3,1),(4,3,2),(4,3,3),(4,3,4),(4,4,1),(4,4,2)\} .
$$

The generation of advice is activated.

Step 4. Choosing different $\delta_{i}$ values, we visualise the corresponding consensus indexes at the decision matrix level of $e_{4}$ in Fig 6, and produce them in Table 1.

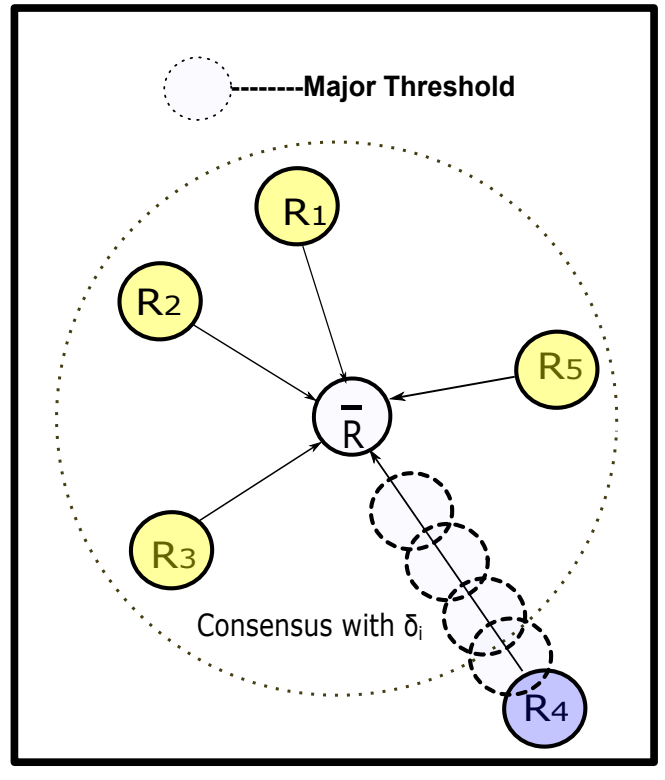

(a) Visual recommendation simulation with $\delta_{i}$

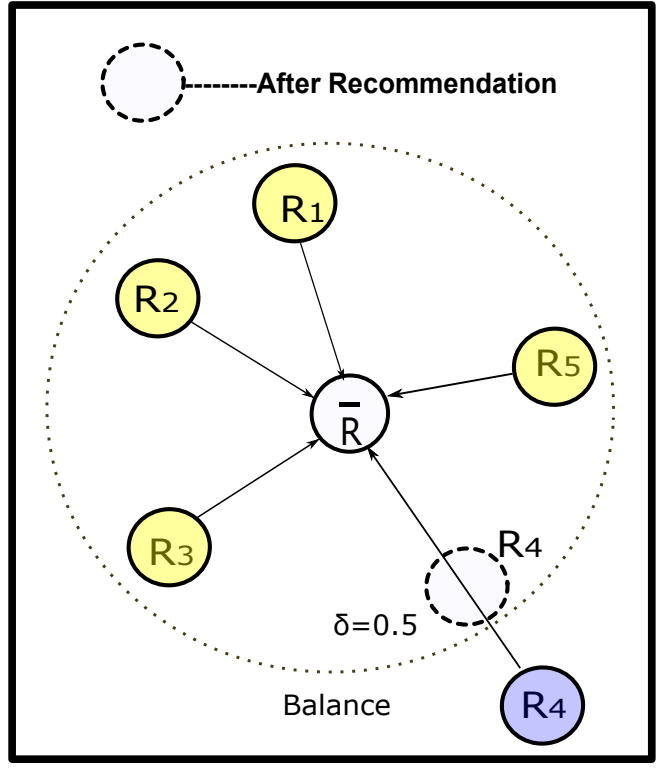

(b) Balance $\delta_{i}$ between individual and group

Figure 6: Visual representation of consensus levels after implementation of recommended values

Table 1: Consensus index at decision matrix level for different $\delta_{i}$ values

\begin{tabular}{l|ccccccccccc}
\hline$\delta$ & 0 & 0.1 & 0.2 & 0.3 & 0.4 & 0.5 & 0.6 & 0.7 & 0.8 & 0.9 & 1.0 \\
\hline$C I$ & 0.860 & 0.870 & 0.878 & 0.888 & 0.897 & 0.906 & 0.915 & 0.924 & 0.934 & 0.943 & 0.952 \\
\hline
\end{tabular}


Obviously, for $\delta \geq 0.5$ the new consensus index is above the consensus threshold value 0.9 is reached. Therefore, the minimum value that will place expert $e_{4}$ above the consensus threshold with lower change variation from his/her original assessment will be $\delta=0.5$. If such value of parameter $\delta$ is chosen by expert $e_{4}$, the system will generate the following recommended values to implement:

To increase your consensus with the rest of the group, your trust assessment for alternative

- $x_{1}$ under criteria $c_{1}$ should to be closer to $(0.539,0.447)$.

- $x_{1}$ under criteria $c_{2}$ should to be closer to $(0.561,0.582)$.

- $x_{1}$ under criteria $c_{3}$ should to be closer to $(0.474,0.579)$.

- $x_{1}$ under criteria $c_{4}$ should to be closer to $(0.539,0.247)$.

- $x_{3}$ under criteria $c_{1}$ should to be closer to $(0.305,0.546)$.

- $x_{3}$ under criteria $c_{2}$ should to be closer to $(0.440,0.638)$.

- $x_{3}$ under criteria $c_{3}$ should to be closer to $(0.631,0.440)$.

- $x_{3}$ under criteria $c_{4}$ should to be closer to $(0.679,0.588)$.

- $x_{4}$ under criteria $c_{1}$ should to be closer to $(0.279,0.696)$.

- $x_{4}$ under criteria $c_{2}$ should to be closer to $(0.379,0.587)$.

Step 5. Once expert $e_{4}$ changes his/her trust decision matrix with the above recommended values, a new consensus process round takes place. The resultant new collective trust decision making matrix is

$$
\overline{\bar{R}}=\left(\begin{array}{cccc}
(0.470,0.388) & (0.404,0.561) & (0.445,0.667) & (0.470,0.414) \\
(0.487,0.690) & (0.482,0.378) & (0.513,0.520) & (0.551,0.641) \\
(0.423,0.485) & (0.371,0.568) & (0.552,0.597) & (0.541,0.461) \\
(0.367,0.577) & (0.467,0.459) & (0.483,0.574) & (0.522,0.583)
\end{array}\right)
$$

The new consensus indexes would become: $C I^{1}=0.921, C I^{2}=0.923, C I^{3}=0.912, C I^{4}=$ 0.906, $C I^{4}=0.903$, which are all above the threshold value $\gamma=0.9$. This means the selection process is activated to derive the group solution of consensus.

Step 6. The criteria weighting vector $\omega=(0.25,0.40,0.15,0.20)^{T}$ produces the following collective overall evaluation values of alternatives:

$$
\bar{r}_{1}=(0.440,0.504), \bar{r}_{2}=(0.502,0.530), \bar{r}_{3}=(0.445,0.530), \bar{r}_{4}=(0.456,0.531)
$$

Their corresponding trust degrees $T D_{1}=0.472, T D_{2}=0.516, T D_{3}=0.487, T D_{4}=0.493$ lead to the final ordering $x_{2} \succ x_{4} \succ x_{3} \succ x_{1}$, which makes alternative $x_{2}$ to be selected as the group solution of consensus. 


\section{Conclusion}

In this article, a novel visual interactive method for consensus in SN-GDM has been presented. This method consists of three main modules: a dual trust propagation, a trust based recommendation and a visual adoption mechanism. The following main advantages with respect to other consensus models proposed in the literature are highlighted:

1. The novel dual trust propagation operator is based on t-norms and t-conorms (Einstein product and Einstein sum used as example in this paper), respectively. It propagates trust in a reasonable way: trust values decrease while distrust values increase. Therefore, in comparison with previous trust propagation operators [31, 38, 41], information attenuation caused by the trusted third parters (TTP) is appropriately addressed and modelled.

2. The trust based recommendation produces advices for inconsistent experts to increase their respective consensus level. The propagated trust by TTP is used to build the trust relationship within the group of experts and, in turn, to obtain their corresponding trust score (TS). TS reflects the actual reputation of experts that derived from historic interaction information in the Social Network, and it can be regarded as a reliable source to assign their corresponding importance degrees. The recommendation advice is thus generated in a reasonable way: the higher the trust score of an expert, the more important the opinion associated to that expert is. Trust relationship between experts in a group is fully exploited to produce recommendations to experts while existing consensus models neglect this usage [14, 44]. Hence, the trust based recommendation is more appropriate to deal with SN-GDM.

3. The reaching consensus visual adoption mechanism supports inconsistent experts by providing them with visual representations of current and future consensus positions before and after recommendation advices are implemented. In the light of this visual extra information, they can see how to reach the consensus threshold value with minimum changes cost, and consequently a balance between group consensus and individual independence is possible to be achieved. Therefore, the advantage of the proposed visual adoption mechanism is that inconsistent experts might be more willing to implement the recommendation advices to increase consensus without being forced to, an aspect existing consensus models do not appropriately address [14, 44].

Although this visual interaction model includes a consensus simulation with different feedback parameters to allow experts to reach consensus, it is not able, however, to determine the exact value of the parameter $\delta$ that would provide the optimal balance between group consensus and individual independence, i.e. the minimum change of original opinion required to contribute to consensus at the threshold value established a priori by the whole group of experts. This is an aspect that deserves further work and that we intent to address in future. 


\section{Acknowledgements}

The authors would like to acknowledge the anonymous referees for providing constructive comments and suggestions. Also acknowledge is given to the financial support received from National Natural Science Foundation of China (NSFC) (No.71571166); Zhejiang Provincial Natural Science Foundation of China (No.LY15G010003); Zhejiang Provincial Philosophy and Social Science Foundation-Zhijiang Young Talent of Social Science (No.16ZJQN046YB); and FEDER funds from projects TIN2013-40658$\mathrm{P}$ and TIN2016-75850-R.

\section{References}

[1] Alonso, S, Prez, I. J., Cabrerizo, F. J. and Herrera-Viedma, E. (2013). A Linguistic Consensus Model for Web 2.0 Communities. Applied Soft Computing 13 (1), 149-157.

[2] Cabrerizo, F. J, Chiclana, F., Al-Hmouz, R., Morfeq, A., Balamash, A. S. and Enrique HerreraViedma (2015) Fuzzy decision making and consensus: challenges. Journal of Intelligent \& Fuzzy Systems. 29 (3), 1109-1118.

[3] Dalal, R. S., and Bonaccio, S. (2010). What types of advice do decision-makers prefer? Organizational Behavior and Human Decision Processes 112, 11-23.

[4] De Cock, M. and Pinheiro Da Silva, P.(2006). A many-valued representation and propagation of trust and distrust. Lecture Notes in Computer Science 3849, 108-113.

[5] Dong, Y. C., and Herrera-Viedma, E. (2015). Consistency-driven automatic methodology to set interval numerical scales of 2-tuple linguistic term sets and its use in the linguistic GDM with preference relation. IEEE Transactions on Cybernetics 45, 780-792.

[6] Dong, Y. C., Zhang, H. J., and Herrera, F. (2015). Minimizing adjusted simple terms in the consensus reaching process with hesitant linguistic assessments in group decision making. Information Sciences 297, 95-117.

[7] Dong, Y. C, Zhang, H. J. and Herrera-Viedma, E. (2016). Integrating experts' weights generated dynamically into the consensus reaching process and its applications in managing non-cooperative behaviors. Decision Support Systems 84, 1-15.

[8] Dong, Y. C., Fan, Z. P., and Yu, S. (2015). Consensus building in a local context for the AHPGDM with the individual numerical scale and prioritization method. IEEE Transactions on Fuzzy Systems 23, 354-368.

[9] Eklund, P., Rusinowska, A. and Swart, H. D. (2008) A consensus model of political decisionmaking. Annals of Operations Research 158 5-20. 
[10] Gong, Z. W, Zhang, H. H., Forrest, J., Li, L. S., and Xu, X. X. (2015). Two consensus models based on the minimum cost and maximum return regarding either all individuals or one individual. Europen Journal of Operational Research 24, 72-83.

[11] Gong, Z. W, Xu, C., Chiclana, F., and Xu, X. X. (2016). Consensus measure with multi-stage fluctuation utility based on China's urban demolition negotiation. Group Decision and Negotiation doi: 10.1007/s10726-016-9486-6.

[12] González-Arteaga, T., de Andrés Calle, R. , and Chiclana, F. (2016) A new measure of consensus with fuzzy preference relations: The correlation consensus degree. Knowledge-Based Systems 10\%, $104-116$.

[13] Hanneman, R. A., and Riddle, M. (2005). Introduction to social network methods. University of California: Riverside.

[14] Herrera-Viedma, E., Alonso, S., Chiclana, F., and Herrera, F. (2007). A consensus model for group decision making with incomplete fuzzy preference relations. IEEE Transactions on Fuzzy Systems 15(5), 863-877.

[15] Herrera-Viedma, E., Chiclana, F., Herrera, F., and Alonso, S. (2007). Group decision-making model with incomplete fuzzy preference relations based on additive consistency. IEEE Transactions on Systems, Man, and Cybernetics, Part B: Cybernetics 37(1), 176-189.

[16] Herrera-Viedma, E., Cabrerizo, F. J., Kacprzyk, J., and Pedrycz, W. (2014). A review of soft consensus models in a fuzzy environment. Information Fusion 17, 4-13.

[17] Kacprzyk, J., Zadrozny, S., and Ras, Z. W. (2010) How to support consensus reaching using action rules: a novel approach. International Journal of Uncertainty, Fuzziness and KnowledgeBased Systems 36, 451-470.

[18] Klement, E. P., Mesiar, R., and Pap, E. (2004). Triangular norms. Position paper 1: basic analytical and algebraic properties. Fuzzy Sets and Systems 143, 5-26.

[19] Li, Y. M, and Lai, C. Y. (2014). A social appraisal simulation for online purchase decision support in the micro-blogosphere. Decision Support Systems 59 190-205.

[20] Liu, J. P, Chen, H. Y, X, Q, Zhou, L. G, and Tao, Z. F (2015). Generalized ordered modular averaging operator and its application to group decision making. Fuzzy Sets and Systems 299, $1-125$.

[21] Liu, Y. J., Liang, C. Y., Chiclana, F. and Wu, J (2016). A trust induced recommendation mechanism for reaching consensus in group decision making. Knowledge-Based Systems, doi: 10.1016/j.knosys.2016.12.014 
[22] Pérez, I. J.,Cabrerizo, F. J., and Herrera-Viedma, E. (2010) A Mobile Decision Support System for Dynamic Group Decision Making Problems. IEEE Transactions on Systems, Man and Cybernetics - Part A: Systems and Humans 40, 1244-1256.

[23] Pérez, I. J., Cabrerizo, F. J., Alonso, S. and Herrera-Viedma, E. (2014). A New Consensus Model for Group Decision Making Problems with Non Homogeneous Experts. IEEE Transactions on Systems, Man, and Cybernetics: Systems 44 (4), 494-498.

[24] Pérez, L. G., Mata, F. and Chiclana, F. (2014). Social network decision making with linguistic trustworthiness based induced OWA operators. International Journal of Intelligent Systems 29 (12), 1117-1137.

[25] Pérez, L. G, Mata, F., Chiclana, F., Kou, G. and Herrera-Viedma, E. (2016). Modelling influence in group decision making. Soft Computing 20, 1653-1665.

[26] Pérez-Asurmendi, P., and Chiclana, F. (2014) Linguistic majorities with difference in support. Applied Soft Computing 18, 196-208.

[27] Recio-García, Juan. A., Quijano, L., and Díaz-Agudo, B. (2013). Including social factors in an argumentative model for Group Decision Support. Decision Support Systems 56, 48-55.

[28] Scott, H. P. (2000). Social Network Analysis: A Handbook. Sage Publications Ltd: London.

[29] Ureña, R., Chiclana, F., Fujita, H., and Herrera-Viedma, E. (2015). Confidence-consistency driven group decision making approach with incomplete reciprocal intuitionistic preference relations. Knowledge-Based Systems 89, 86-96.

[30] Ureña, R., Chiclana, F., Morente, J. A., and Herrera-Viedma, E. (2014). Managing incomplete preference relations in decision making: a review and future trends. Information Sciences 302, $14-32$.

[31] Victor, P., Cornelis, C., De Cock, M., and Herrera-Viedma., E. (2011). Practical aggregation operators for gradual trust and distrust. Fuzzy Sets and Systems 184(1), 126-147.

[32] Victor, P., Cornelis, C., De Cock, M., and Pinheiro da Silva., P. (2009). Gradual trust and distrust in recommender systems. Fuzzy Sets and Systems 160(10), 1367-1382.

[33] Wasserman, S., and Faust, K. (2009). Social Network Analysis: Methods and Applications. Cambridge University Press.

[34] Wu, J., and Chiclana, F. (2014). Visual information recommendation simulation and attitudinal prioritisation method for group decision making with triangular fuzzy complementary preference relations. Information Sciences 279, 716-736. 
[35] Wu, J. and Chiclana, F. (2014). Multiplicative consistency of intuitionistic reciprocal preference relations and its application to missing values estimation and consensus building. Knowledge-Based Systems 71, 187-200.

[36] Wu, J., and Chiclana, F. (2014). A risk attitudinal ranking method for interval-valued intuitionistic fuzzy numbers based on novel score and accuracy expected functions. Applied Soft Computing 22, 272-286.

[37] Wu, J., and Chiclana, F. (2014). A social network analysis trust-consensus based approach to group decision-making problems with interval-valued fuzzy reciprocal preference relations. Knowledge-Based Systems 59, 97-107.

[38] Wu, J., Chiclana, F., and Herrera-Viedma, E. (2015). Trust based consensus model for social network in an incomplete linguistic information context. Applied Soft Computing 35, 827-839.

[39] Wu, J., Chiclana, F., Liao, H. C (2016) Isomorphic multiplicative transitivity for intuitionistic and interval-valued fuzzy preference relations and its application in deriving their priority vectors. IEEE Transactions on Fuzzy Systems doi: 10.1109/TFUZZ.2016.2646749.

[40] Wu, J., Liu, Y. J., and Liang, C. Y. (2015). A consensus- and harmony-based feedback mechanism for multiple attribute group decision making with correlated intuitionistic fuzzy sets. International Transactions in Operational Research 22, 1033-1054.

[41] Wu, J., Xiong, R. Y., and Chiclana, F. (2016). Uninorm trust propagation and aggregation methods for group decision making in social network with four tuple information. Knowledge-Based Systems 96, 29-39.

[42] Xia, M. M., and Chen, J. (2015) Multi-criteria group decision making based on bilateral agreements. European Journal of Operational Research 240, 756-764.

[43] Xu, J., Wan, S. P., and Dong, J. Y. (2016). Aggregating decision information into Atanassov's intuitionistic fuzzy numbers for heterogeneous multi-attribute group decision making. Applied Soft Computing 41, 331-351.

[44] Xu, J. P, and Wu, Z. B. (2013). A maximizing consensus approach for alternative selection based on uncertain linguistic preference relations. Computers and Industrial Engineering 64, 999-1008.

[45] Yager, R. R. (1996). Quantifier guided aggregation using OWA operators. International Journal of Intelligent Systems 11, 49-73.

[46] Yager, R. R., and Filev, D. P. (1999). Induced ordered weighted averaging operators. IEEE Transactions on Systems, Man and Cybernetics 29, 141-150. 
[47] Yager, R. R., and Alajlan, N. (2015) An intelligent interaction approach to group aggregation of subjective probabilities. Knowledge-Based Systems 58, 3-10.

[48] Zhang, X. X., Ge, B. F., Jian, J., and Tan, Y. J (2016). Consensus building in group decision making based on multiplicative consistency with incomplete reciprocal preference relations. Knowledge-Based Systems 106, 96-104. 